\title{
Curcumin protects against acute renal injury by suppressing JAK2/STAT3 pathway in severe acute pancreatitis in rats
}

\author{
SHUAIJUN ZHU ${ }^{1 *}$, CHI ZHANG ${ }^{2 *}$, QINYONG WENG ${ }^{3}$ and BING YE ${ }^{1}$ \\ ${ }^{1}$ Intensive Care Unit, Fujian Medical University Union Hospital; ${ }^{2}$ Intensive Care Unit, Fujian Provincial Hospital; \\ ${ }^{3}$ Department of Geriatrics, Fujian Medical University Union Hospital, Fuzhou, Fujian 350001, P.R. China
}

Received April 25, 2016; Accepted March 17, 2017

DOI: 10.3892/etm.2017.4647

\begin{abstract}
The aim of the present study was to investigate the effect of curcumin on acute renal injury in a rat model of severe acute pancreatitis (SAP). A SAP model with acute kidney injury was established in rats by retrograde injection of $5 \%$ sodium taurocholate into the pancreatic duct. The serum amylase, creatinine ( $\mathrm{Cr}$ ) and blood urea nitrogen (BUN) levels in rats were measured. Hematoxylin and eosin staining was used to assess pancreatic and renal histological changes. Serum tumor necrosis factor (TNF)- $\alpha$ and interleukin (IL)-6 levels were measured using ELISA kits. Renal protein levels of Janus kinase (JAK) 2/signal transducer and activator of transcription (STAT) 3 pathway components were determined by western blot assay. The results showed that curcumin significantly decreased serum amylase, Cr and BUN levels, and alleviated pancreatic and renal histological changes in SAP rats. Furthermore, curcumin markedly decreased serum TNF- $\alpha$ and IL- 6 levels and downregulated renal protein levels of JAK2/STAT3 pathway components. These results proved that curcumin ameliorates acute renal injury in a rat model of SAP. The molecular mechanism of its effect may be associated with the suppression of the JAK2/STAT3 pathway to reduce TNF- $\alpha$ and IL- 6 levels in SAP-induced acute renal injury. Therefore, the findings of the present study revealed the potential use of curcumin for the prevention and treatment of SAP and the associated renal injury.
\end{abstract}

\section{Introduction}

Severe acute pancreatitis (SAP), an acute inflammatory condition of the pancreas, is considered to be a paradigm of sterile

Correspondence to: Dr Shuaijun Zhu, Intensive Care Unit, Fujian Medical University Union Hospital, 29 Xinquan Road, Fuzhou, Fujian 350001, P.R. China

E-mail: zhu_shuaijunfz@163.com

*Contributed equally

Key words: severe acute pancreatitis, acute renal injury, curcumin, JAK2, STAT3, inflammation inflammation leading to systemic multiple organ dysfunction syndrome (MODS) and death. Acute renal injury (ARI) is one of the main complications of SAP and significantly increases the mortality rate of patients with AP (66.6 vs. 14.5\%) (1). However, the underlying mechanisms of ARI occurring in patients with SAP have remained to be clarified. Increasing evidence has indicated that pro-inflammatory cytokines, including tumor necrosis factor- $\alpha(\mathrm{TNF}-\alpha)$ and interleukin (IL)-6, have an important role in the pathological mechanisms of SAP and SAP-associated organ failure (2-4). Therefore, inhibiting the transcription and translation of mediators to reduce the secretion of pro-inflammatory factors may ameliorate inflammation and renal failure in SAP.

Signal transducers and activators of transcription (STATs), a protein family comprised of seven members (STAT1, -2, -3, $-4,-5 \mathrm{a},-5 \mathrm{~b}$ and -6$)$, generally transduce signals from activated receptors or intracellular kinases to the nucleus, thus activating and regulating gene transcription (5). The Janus kinase 2 (JAK2)/STAT3 pathway is well known to be involved in the immune response of numerous cytokines, including TNF- $\alpha$ and IL-6 (6). In addition, evidence derived from numerous clinical and experimental studies suggests the involvement of the JAK2/STAT3 pathway in pancreatitis (7-9) or renal diseases (10-12).

Curcumin (diferuloylmethane), the active phytochemical component of turmeric (a spice used mostly in Asia) has been isolated from the rhizome of the Curcuma longa plant. Curcumin has a myriad of pharmacological effects, including anti-inflammatory $(13,14)$, anti-bacterial $(15)$, anti-oxidative (16) and renal-protective activities $(17,18)$. A previous study suggested that curcumin has a therapeutic role in a rat model of SAP (19). Another study has reported that curcumin inhibited renal inflammation in cisplatin-induced nephrotoxicity in mice (20) and suppressed JAK2/STAT3 signaling to ameliorate renal endothelial dysfunction in fructose-fed rats (21). All of the above suggests the potential use of curcumin in the treatment of acute renal failure following SAP. Thus, the present study investigated the effect of curcumin on acute renal failure arising from SAP and to explore the molecular mechanism.

\section{Materials and methods}

Chemicals and reagents. Curcumin and hematoxylin and eosin were purchased from Sigma-Aldrich (Merck KGaA, Darmstadt, 
Germany). ELISA kits for assaying TNF- $\alpha$ and IL- 6 were obtained from R\&D (Minneapolis, MN, USA). Assay kits for amylase (AMY), creatinine $(\mathrm{Cr})$ and blood urea nitrogen (BUN) were purchased from Jiancheng Biotech (Nanjing, China). TRIzol reagent was obtained from Invitrogen (Thermo Fisher Scientific, Inc., Waltham, MA, USA) and Moloney murine leukemia virus (M-MLV) reverse transcriptase was from Promega, Corp. (Madison, WI, USA). Antibodies against JAK2 (cat no. 3230), phosphorylated (p)-JAK2 (cat no. 3776), STAT3 (cat no. 4904), p-STAT3 (cat no. 9145) and suppressor of cytokine signaling 3 (SOCS3; cat no. 2923) were purchased from Cell Signaling Technology, Inc. (Danvers, MA, USA) and horseradish peroxidase (HRP)-conjugated GAPDH antibody (cat no. sc-2577) was purchased from Santa Cruz Biotechnology (Dallas, TX, USA).

Animals. A total of 30 male Sprague-Dawley rats (age, 8-10 weeks; weight, 200-250 g) were obtained from Shanghai Laboratory Animals Co., Ltd. (Shanghai, China). All animals were housed under standardized conditions with a 12-h light/dark cycle with free access to standard laboratory chow and water. All protocols and procedures used in the study complied the US National Institute of Health Guidelines for the Care and Use of Laboratory Animals and were approved by the Institutional Animal Care and Utilization Committee of Fujian Medical University (Fuzhou, China).

Experimental models and groups. The rats were divided into three subgroups: Sham controls, SAP rats treated with saline and SAP rats treated with curcumin $(100 \mathrm{mg} / \mathrm{kg})$. Rats were fasted $12 \mathrm{~h}$ prior to the operation. The three groups were dived into 3, 12 and $24 \mathrm{~h}$ time-point groups (each sub-group contained 3 rats). After anesthesia with $10 \%$ chloraldurat $(0.3 \mathrm{ml} / 100 \mathrm{~g}$, Sigma Aldrich; Merck KGaA, Darmstadt, Germany), the SAP model was induced by a standard pressure-controlled infusion of a freshly prepared 5\% sodium taurocholate (Sigma Aldrich; Merck KGaA) solution $(0.1 \mathrm{ml} / 100 \mathrm{~g})$ into the bile-pancreatic duct under laparotomy as previously described (22). Rats in the sham control group underwent the same operative procedure without the injection of 5\% sodium taurocholate in the pancreatic duct. In the SAP + Cur group, rats were intraperitoneally injected with curcumin solution $(100 \mathrm{mg} / \mathrm{kg}) 30 \mathrm{~min}$ prior to the establishment of the SAP model. After surgery, all animals were subcutaneously injected with saline $(0.2 \mathrm{ml} / \mathrm{kg})$ in order to replenish the missing fluid.

Blood and tissue preparation. The rats were sacrificed at designated time-points after the induction of pancreatitis. The blood samples were obtained via intracardiac puncture and then centrifuged to obtain the serum to be stored at $-20^{\circ} \mathrm{C}$ until assay. Pancreas and kidney tissues were dissected quickly on ice, and parts of tissues were immediately fixed for hematoxylin and eosin staining analysis, while others were frozen in liquid nitrogen and stored at $-80^{\circ} \mathrm{C}$ for later analysis.

Hematoxylin and eosin staining analysis. After blood collection, rat pancreas and kidney tissues were removed, immediately fixed and preserved in $70 \%$ ethanol. Subsequent to embedding in paraffin, specimens were cut in $4-\mu \mathrm{m}$-thick sections and mounted on 3-aminopropyltriethoxysilane-coated glass slides. The sections were de-paraffinized in xylene, re-hydrated in decreasing concentrations of ethanol in water and stained with hematoxylin and eosin reagent. Examination of sections was performed with a light microscope (Olympus Ltd., Tokyo, Japan).

Serum AMY, BUN and Cr assays. Serum levels of AMY and $\mathrm{Cr}$ as well as BUN were measured using standard diagnostic kits following the manufacturer's instructions.

Serum TNF- $\alpha$ and IL- 6 levels. ELISA kits were used to detect serum TNF- $\alpha$ and IL- 6 levels according to the manufacturer's instructions.

Reverse-transcription quantitative polymerase chain reaction $(R T-q P C R)$ analysis. SOCS3 mRNA in the kidney tissue was detected by RT-qPCR analysis. In brief, total RNA was extracted from kidney tissues with TRIzol reagent according to the manufacturer's instructions, and was reverse-transcribed into complementary DNA using an oligo (dT) primer (SunShine Biotechnology, Nanjing, China) and M-MLV reverse transcriptase. The $20 \mu \mathrm{l}$ PCR reaction mixture was prepared and contained 2- $\mu$ l cDNA, $10 \mu 1$ Power SYBR-Green PCR Supermix (Bio-Rad Laboratories, Inc., Hercules, CA, USA) and $1 \mu \mathrm{l}$ of each primer and H2O-DEPC to $20 \mu \mathrm{l}$. RT-qPCR analysis were performed using SYBR-Green I dye (Bio-Rad Laboratories, Inc.) under the following reaction conditions: $1 \mathrm{~min}$ at $95^{\circ} \mathrm{C}$ followed by 40 cycles of $15 \mathrm{sec}$ at $95^{\circ} \mathrm{C}, 1 \mathrm{~min}$ at $60^{\circ} \mathrm{C}$. The following primers were used: SOCS3 forward, $5^{\prime}$-TTCGCC CTTAGCGTGAAGATGG-3' and reverse, 5'-TAGTGCTCC AGCAGCTCGAAGA-3'; GAPDH forward, 5'-CTTTGGTAT CGTGGAAGGACTC-3' and reverse, 5'-GTAGAGGCAGGGA TGATGTTCT-3'. The comparative Cq $\left(2^{-\Delta \Delta C q}\right)$ method was used to analyze the relative gene expression levels, as previously described (23). GAPDH was used as an internal positive control.

Western blot analysis. The expression of JAK2, STAT3 and SOCS3 protein as well as the levels of p-JAK2 and p-STAT3 in kidney tissues were measured by western blot analysis. Protein extracts were obtained by homogenizing samples in a cell lysis buffer (10 mM Tris-HCl, $1 \mathrm{mM}$ EDTA and $250 \mathrm{mM}$ sucrose, $\mathrm{pH} 7.4$, containing $15 \mu \mathrm{g} / \mathrm{ml}$ aprotinin, $5 \mu \mathrm{g} / \mathrm{ml}$ leupeptin, $0.1 \mathrm{mM}$ phenylmethanesulfonyl fluoride, $1 \mathrm{mM} \mathrm{NaF}$ and $1 \mathrm{mM} \mathrm{Na} \mathrm{VO}_{4}$ ), followed by centrifugation at 12,000 x g for $15 \mathrm{~min}$. The protein concentration was determined using a bicinchoninic acid kit (Thermo Fisher Scientific, Inc.). A total of $30 \mu \mathrm{g}$ protein per lane was separated by $10 \%$ SDS-PAGE, transferred onto polyvinylidene fluoride membranes (Millipore, Billerica, MA, USA), blocked with 5\% skim milk and incubated with primary antibodies (p-JAK2: 1:1,000, JAK2: 1:1,000, p-STAT3, 1:1,000, SOCS3: 1:1,000, GAPDH: $1: 500)$ at $4^{\circ} \mathrm{C}$ overnight. Then the blots were incubated with the corresponding secondary antibody (HRP-conjugated whole-goat anti-rabbit IgG, 1:10,000; cat no. 074-1506; Kirkegaard \& Prerry Lab Inc., Gaithersburg, MD, USA) for $2 \mathrm{~h}$ at room temperature. Finally, immunoreactive bands were visualized via enhanced chemiluminescence (Cell Signaling Technology, Inc., Danvers, MA, USA) and the membranes were then immediately exposed to autoradiographic film (Eastman Kodak, Rochester, NY, USA). 

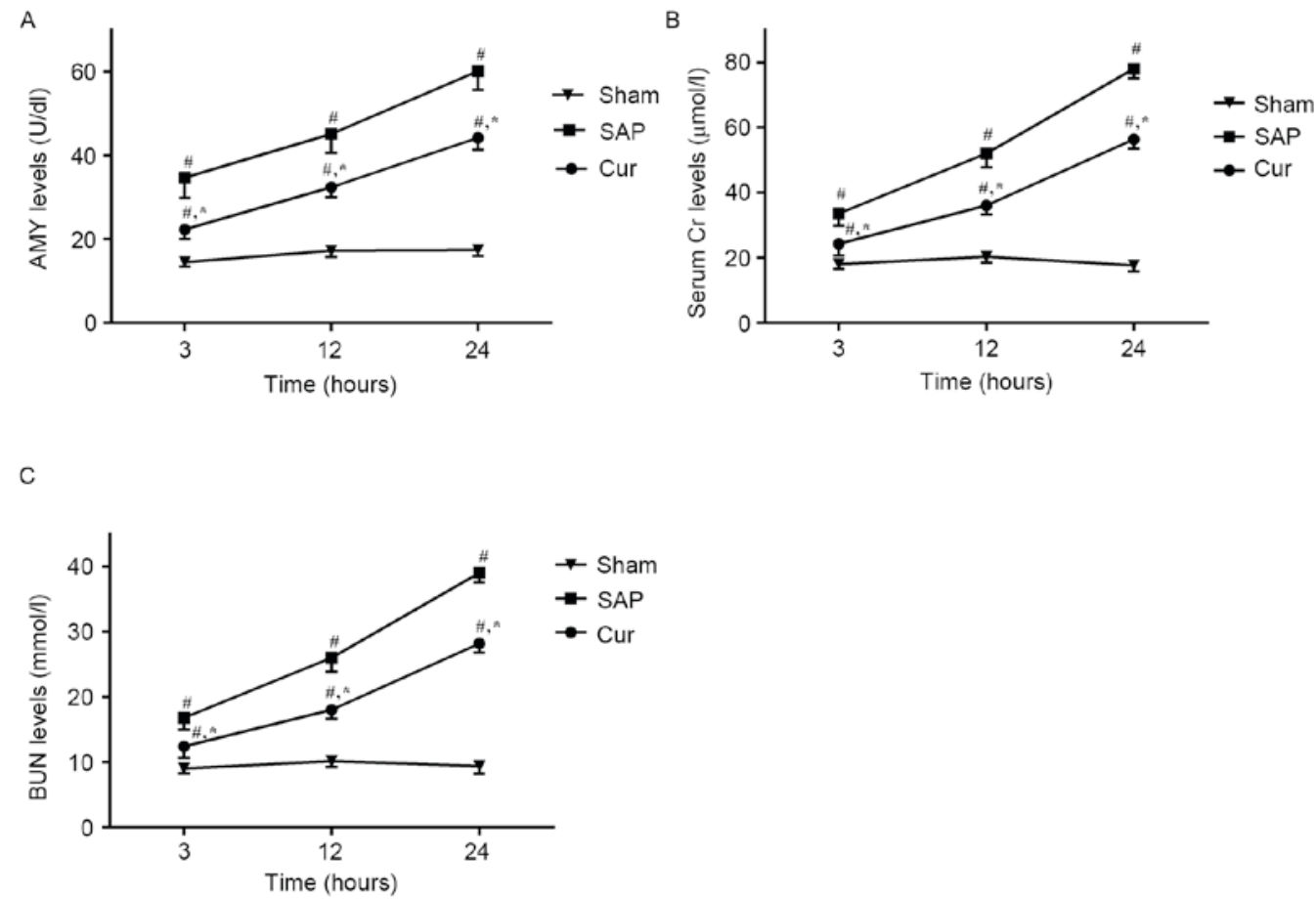

Figure 1. Effects of curcumin on the levels of serum (A) AMY and (B) $\mathrm{Cr}$ as well as (C) BUN in SAP rats. Values are expressed as the mean \pm standard deviation $(\mathrm{n}=4) .{ }^{~} \mathrm{P}<0.05$ vs. Sham group; ${ }^{*} \mathrm{P}<0.05$ vs. SAP group. SAP, severe acute pancreatitis; Cur, SAP model rats pre-treated with curcumin; $\mathrm{Cr}$, creatinine; BUN, blood urea nitrogen; AMY, amylase.

Statistical analysis. Results were expressed as the mean \pm standard deviation. Statistical analysis was performed using one-way analysis of variance followed by a post-hoc test (Fisher's least significant differences test) as appropriate with the statistical analysis system GraphPad Prism 6 (GraphPad Software, Inc., La Jolla, CA, USA). $\mathrm{P}<0.05$ was considered to indicate a statistically significant difference.

\section{Results}

Curcumin attenuates $S A P$-induced increases in serum AMY, $\mathrm{Cr}$ and BUN. As shown in Fig. 1A, serum AMY levels were significantly increased in the SAP group at all time-points in comparison with those in the Sham group. However, in the Cur group, these increases in serum AMY levels were significantly inhibited. Moreover, the SAP group showed elevated levels of serum Cr (Fig. 1B) and BUN (Fig. 1C) compared with those in the Sham group. Treatment with curcumin obviously decreased the levels of serum $\mathrm{Cr}$ and BUN at the respective time-points.

Curcumin pre-treatment reduces histopathological changes in the pancreas and kidneys of SAP rats. Pancreatic and renal histological changes were assessed using hematoxylin and eosin staining. As shown in Fig. 2, obvious edema, inflammatory cell infiltration and necrosis were observed in the pancreases of SAP rats at $12 \mathrm{~h}$ (Fig. 2B). Compared to the SAP group, the pancreatic histological injuries were significantly ameliorated by pre-treatment with curcumin (Fig. 2C). Furthermore, Fig 3 shows that, SAP rats at $12 \mathrm{~h}$ demonstrated glomerular and tubular damage as well as inflammatory cell infiltration, suggesting renal injury (Fig. 3B). However, rats pre-treated with curcumin demonstrated reduced histological features in the kidney in comparison with those in the SAP rats (Fig. 3C).

Curcumin reduces SAP-induced increases of serum TNF- $\alpha$ and IL-6 levels in SAP rats. The effect of curcumin on the serum levels of inflammatory cytokines, TNF- $\alpha$ and IL-6, were analyzed using ELISA kits. As shown in Fig. 4, a substantial increase in TNF- $\alpha$ and IL- 6 levels was observed in the SAP group. However, pre-treatment with curcumin significantly decreased serum levels of TNF- $\alpha$ and IL- 6 in this animal model.

Curcumin suppresses the JAK2/STAT3 signaling pathway in SAP rats. Elevated mRNA and protein levels of SOCS3 were observed in the kidneys of SAP rats. These alterations in SAP rats were attenuated by pre-treatment with curcumin at the mRNA and protein level (Fig. 5A and B). To further determine JAK and STAT3 signaling in the kidneys of rats at $12 \mathrm{~h}$ after induction of SAP, western blot analysis was applied. Compared to the Sham group, the SAP group showed elevated renal protein levels of $\mathrm{p}-\mathrm{JAK} 2$ and $\mathrm{p}$-STAT3. Curcumin distinctly inhibited the activation of JAK2 and STAT3 in this model (Fig. 5B).

\section{Discussion}

The present study demonstrated that a) renal injury caused by SAP was significantly improved by pre-treatment with curcumin, b) curcumin greatly decreased the secretion of inflammatory cytokines and c) the mechanism by which curcumin ameliorates renal injury includes the reduction 

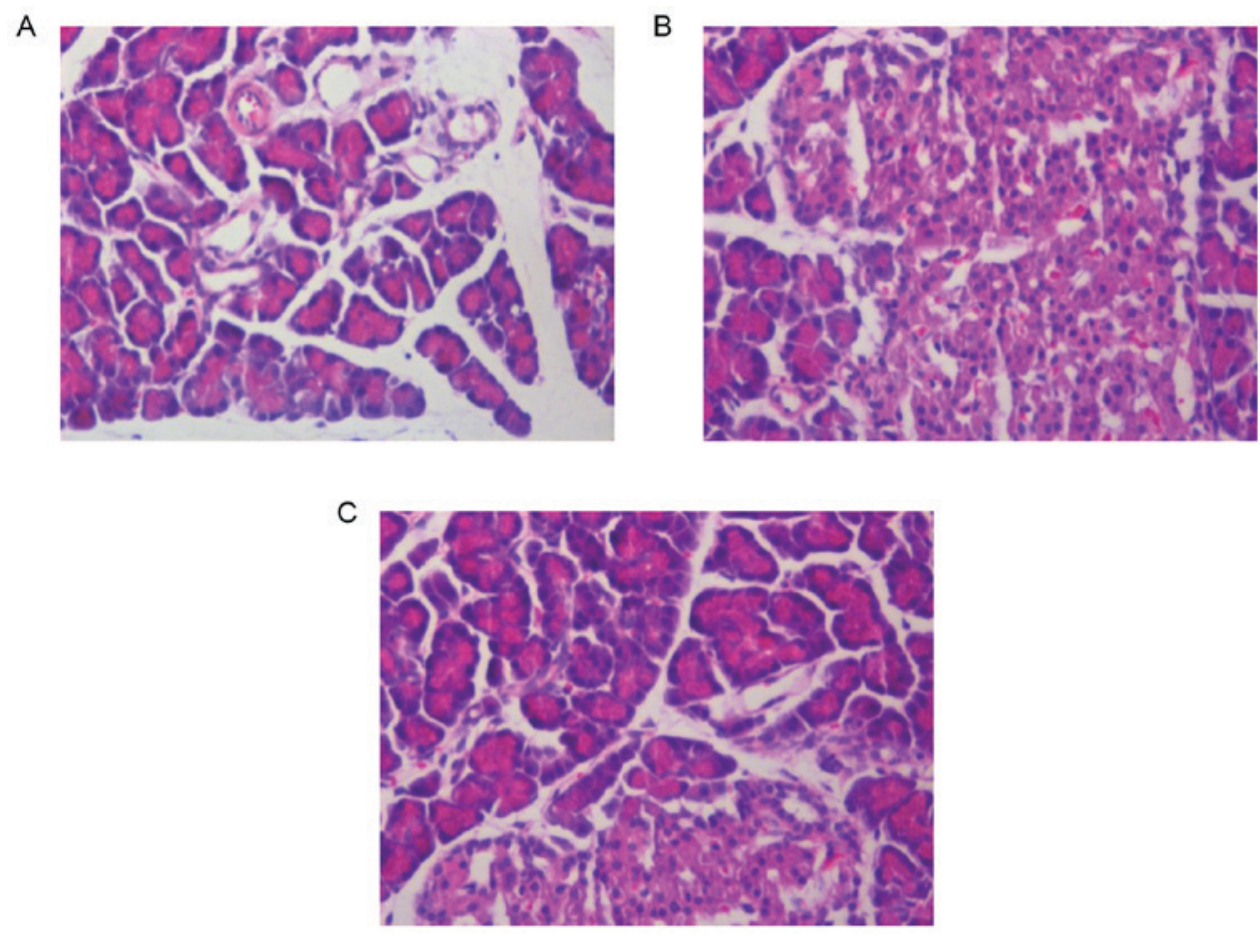

Figure 2. Effects of curcumin on morphological changes in the pancreas at $12 \mathrm{~h}$ following SAP in rats. Hematoxylin and eosin staining of pancreatic tissue of (A) Sham group, (B) SAP group and (C) curcumin-pre-treated SAP model rats (magnification, x200). SAP, severe acute pancreatitis.
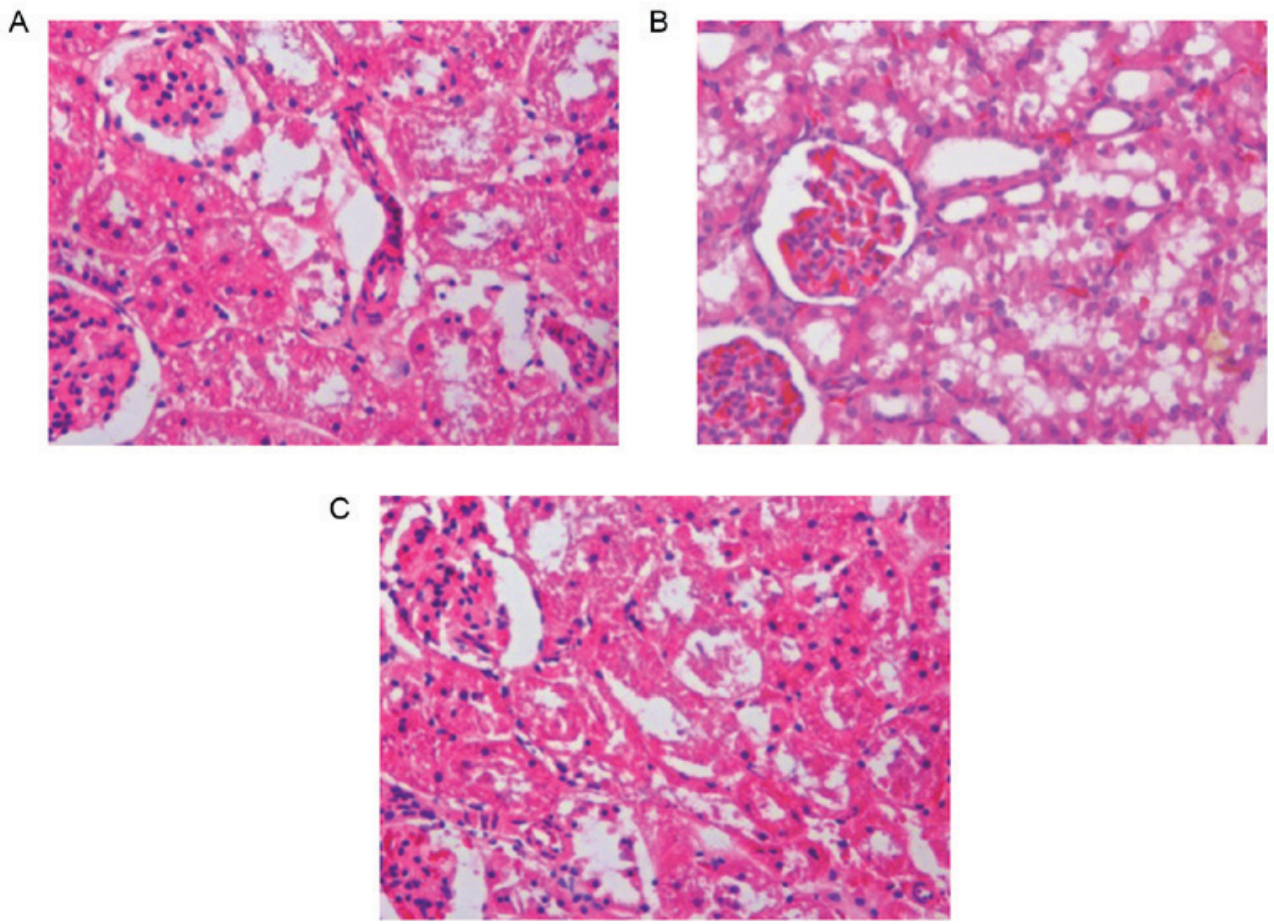

Figure 3. Effects of curcumin on morphological changes in the kidney at $12 \mathrm{~h}$ following SAP in rats. Hematoxylin and eosin staining of the renal tissue of (A) Sham group, (B) SAP group, (C) curcumin-pre-treated SAP model rats (magnification, x200). SAP, severe acute pancreatitis.

of inflammation and suppression of JAK2/STAT3 pathway activation.

A previous study confirmed the protective effect of curcumin in a rat model of SAP (19). In the present study, hyperamylasemia and pancreatic pathological evidence were observed in the SAP model group, which were obviously improved by pre-treatment with curcumin. Moreover, the levels of BUN and serum creatinine were increased, alongside obvious pathological damage, which was indicative of renal injury in this SAP model. However, pre-treatment with curcumin significantly ameliorated the pathological changes in the kidneys of SAP rats and also decreased BUN 
A

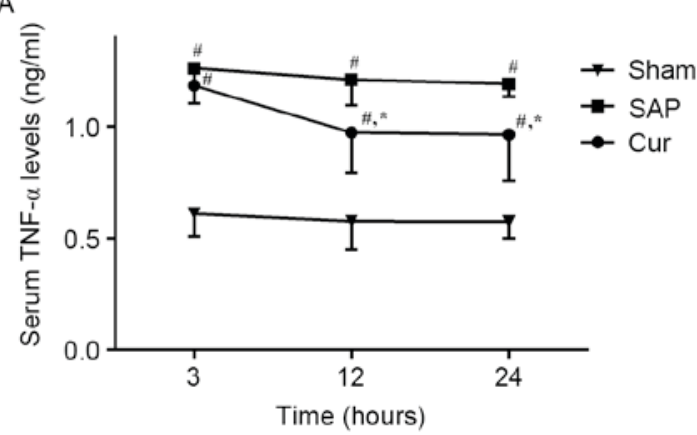

B

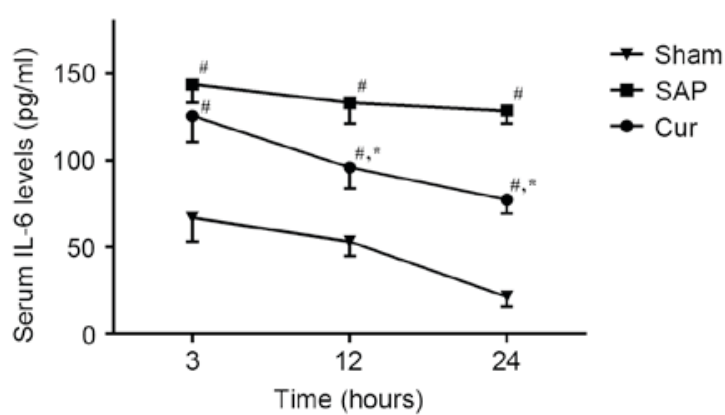

Figure 4. Effects of curcumin on serum (A) TNF- $\alpha$ and (B) IL-6 levels in SAP rats. Values are expressed as the mean \pm standard deviation (n=4). ${ }^{\#} \mathrm{P}<0.05$ vs. Sham group; "P $<0.05$ vs. SAP group. SAP, severe acute pancreatitis; Cur, SAP model rats pre-treated with curcumin; TNF, tumor necrosis factor; IL, interleukin.

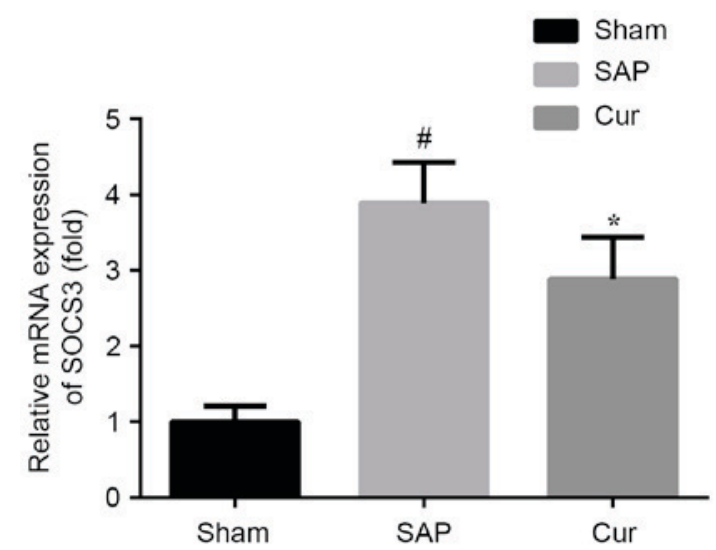

B

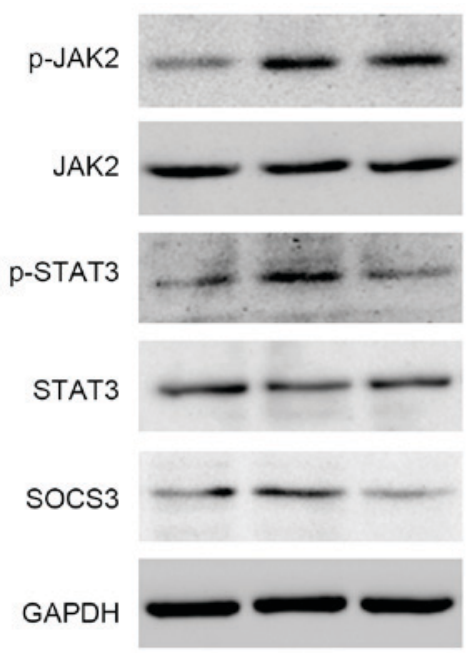

Figure 5. Effects of curcumin on (A) SOCS3 mRNA and (B) protein levels of JAK2/STAT3 pathway components in SAP rats. Values are expressed as the mean \pm standard deviation $(\mathrm{n}=4)$. ${ }^{\mathrm{P}} \mathrm{P}<0.05$ vs. Sham group; ${ }^{\mathrm{P}}<0.05$ vs. SAP group. SAP, severe acute pancreatitis; Cur, SAP model rats pre-treated with curcumin; p-JAK2, phosphorylated Janus kinase 2; STAT3, signal transducer and activator of transcription 3; SOCS3, suppressor of cytokine signaling 3.

and serum $\mathrm{Cr}$ levels in rats with renal injury associated with SAP.

At present, the pathogenesis of SAP-induced renal injury remains elusive. It has been generally recognized that the excess release of cytokines and inflammatory mediators has a pivotal role in SAP and SAP-associated renal injury (24). Cytokines such as IL-6 and TNF- $\alpha$ exert a major effect on the outcome of SAP, particularly by triggering the systemic inflammatory response and multisystem organ failure, which is mostly responsible for the associated mortality (25). In the present study, increased serum levels of IL- 6 and TNF- $\alpha$ were observed in the SAP group, which were greatly decreased by treatment with curcumin. These results combined with the histological and biochemical changes in the kidneys of SAP rats indicated that curcumin attenuated renal injury in SAP rats, at least in part via the inhibition of inflammatory cytokines.

The JAK/STAT pathway is a pleiotropic cascade essential to cytokine and growth hormone receptor signaling (26). An increasing body of evidence has suggested the involvement of the JAK/STAT pathway in renal disease (27-29). A previous study indicated that the activation of JAK/STAT3 signaling induces the expression of IL-1 $\beta$ in cerulean-stimulated pancreatic acinar cells (30). Of note, studies have shown that curcumin, as an inhibitor of the JAK2/STAT3 pathway, exerts marked effects in several experimental models and human diseases $(21,31)$. The present study found that the phosphorylation of JAK2 and STAT3 was significantly increased in the kidneys of SAP rats, which was inhibited by pre-treatment with curcumin, suggesting that the inflammatory response triggered by SAP was effectively inhibited by curcumin via interfering with JAK2/STAT3 pathway activation. However, few studies have investigated the role of JAK2/STAT3 signaling in renal injury following SAP. The present study provided in vivo evidence that the JAK2/STAT3 pathway is activated in renal injury following SAP, and that the renoprotective effect of curcumin in SAP is associated with the inhibition of this activation.

In conclusion, the present study demonstrated the renoprotective effect of curcumin in a rat model of SAP. The underlying mechanism of its effect is in part the inhibition of JAK2/STAT3 pathway activation to reduce the inflammation cascade and inflammatory cytokine secretion. Therefore, the findings of the present study revealed the potential use of 
curcumin for the prevention and treatment of SAP and the associated renal injury.

\section{Acknowledgements}

The present study was supported by a grant from the Geriatric Key Clinical Specialty Discipline Construction Programs of China and Fujian Province (2013).

\section{References}

1. Li H, Qian Z, Liu Z, Liu X, Han X and Kang H: Risk factors and outcome of acute renal failure in patients with severe acute pancreatitis. J Crit Care 25: 225-229, 2010.

2. Papachristou GI, Clermont G, Sharma A, Yadav D and Whitcomb DC: Risk and markers of severe acute pancreatitis. Gastroenterol Clin North Am 36: 277-296, viii, 2007.

3. Zhang XP, Wang L and Zhou YF: The pathogenic mechanism of severe acute pancreatitis complicated with renal injury: A review of current knowledge. Digest Dis Sci 53: 297-306, 2008.

4. Zhu AJ, Shi JS and Sun XJ: Organ failure associated with severe acute pancreatitis. World J Gastroentero 9: 2570-2573, 2003.

5. Rawlings JS, Rosler KM and Harrison DA: The JAK/STAT signaling pathway. J Cell Sci 117: 1281-1283, 2004.

6. Yang X, He G, Hao Y, Chen C, Li M, Wang Y, Zhang G and $\mathrm{Yu} \mathrm{Z}$ : The role of the JAK2-STAT3 pathway in pro-inflammatory responses of EMF-stimulated N9 microglial cells. J Neuroinflammation 7: 54, 2010.

7. $\mathrm{Yu} \mathrm{JH}$ and Kim H: Role of janus kinase/signal transducers and activators of transcription in the pathogenesis of pancreatitis and pancreatic cancer. Gut Liver 6: 417-422, 2012.

8. Vona-Davis LC, Frankenberry KA, Waheed U, Peterson E and McFadden DW: Expression of STAT3 and SOCS3 in pancreatic acinar cells 1,2. J Surg Res 127: 14-20, 2005.

9. Ju KD, Lim JW, Kim KH and Kim H: Potential role of NADPH oxidase-mediated activation of Jak2/Stat 3 and mitogen-activated protein kinases and expression of TGF- $\beta 1$ in the pathophysiology of acute pancreatitis. Inflamm Res 60: 791-800, 2011.

10. Horiguchi A, Asano T, Kuroda K, Sato A, Asakuma J, Ito K, Hayakawa M, Sumitomo M and Asano T: STAT3 inhibitor WP1066 as a novel therapeutic agent for renal cell carcinoma. Brit J Cancer 102: 1592-1599, 2010.

11. Brands MW, Banes-Berceli AK, Inscho EW, Al-Azawi H, Allen AJ and Labazi H: Interleukin 6 knockout prevents angiotensin II hypertension: role of renal vasoconstriction and janus kinase 2/signal transducer and activator of transcription 3 activation. Hypertension 56: 879-884, 2010

12. Pang M, Ma L, Gong R, Tolbert E, Mao H, Ponnusamy M, Chin YE, Yan H, Dworkin LD and Zhuang S: A novel STAT3 inhibitor, S3I-201, attenuates renal interstitial fibroblast activation and interstitial fibrosis in obstructive nephropathy. Kidney Int 78: 257-268, 2010.

13. Aggarwal BB and Harikumar KB: Potential therapeutic effects of curcumin, the anti-inflammatory agent, against neurodegenerative, cardiovascular, pulmonary, metabolic, autoimmune, and neoplastic diseases. Int J Biochem Cell Biol 41: 40-59, 2009.

14. Wang ME, Chen YC, Chen IS, Hsieh SC, Chen SS and Chiu $\mathrm{CH}$ : Curcumin protects against thioacetamide-induced hepatic fibrosis by attenuating the inflammatory response and inducing apoptosis of damaged hepatocytes. J Nutr Biochem 23: 1352-1366, 2012.
15. Mun SH, Joung DK, Kim YS, Kang OH, Kim SB, Seo YS Kim YC, Lee DS, Shin DW, Kweon KT and Kwon DY: Synergistic antibacterial effect of curcumin against methicillin-resistant Staphylococcus aureus. Phytomedicine 20: 714-718, 2013.

16. Kim BH, Lee ES, Choi R, Nawaboot J, Lee MY, Lee EY, Kim HS and Chung $\mathrm{CH}$ : Protective effects of curcumin on renal oxidative stress and lipid metabolism in a rat model of Type 2 diabetic nephropathy. Yonsei Med J 57: 664-673, 2016.

17. Trujillo J, Chirino YI, Molina-Jijón E, Andérica-Romero AC, Tapia E and Pedraza-Chaverrí J: Renoprotective effect of the antioxidant curcumin: Recent findings. Redox Biol 1: 448-456, 2013.

18. Karahan MA, Yalcin S, Aydogan H, Büyükfirat E, Kücük A Kocarslan S, Yüce HH, Taskın A and Aksoy N: Curcumin and dexmedetomidine prevents oxidative stress and renal injury in hind limb ischemia/reperfusion injury in a rat model. Ren Fail 38: 693-698, 2016.

19. Zhong K: Curcumin mediates a protective effect via TLR-4/NF- $\kappa \mathrm{B}$ signaling pathway in rat model of severe acute pancreatitis. Cell Biochem Biophys 73: 175-180, 2015.

20. Ueki M, Ueno M, Morishita J and Maekawa N: Curcumin ameliorates cisplatin-induced nephrotoxicity by inhibiting renal inflammation in mice. J Biosci Bioeng 115: 547-551, 2013.

21. Zhang DM, Li YC, Xu D, Ding XQ and Kong LD: Protection of curcumin against fructose-induced hyperuricaemia and renal endothelial dysfunction involves NO-mediated JAK-STAT signalling in rats. Food Chem 134: 2184-2193, 2012.

22. Zhang XH, Li ML, Wang B, Guo MX and Zhu RM: Caspase-1 inhibition alleviates acute renal injury in rats with severe acute pancreatitis. World J Gastroenterol 20: 10457-10463, 2014.

23. Livak KJ and Schmittgen TD: Analysis of relative gene expression data using real-time quantitative PCR and the 2(-Delta Delta C(T)) Method. Methods 25: 402-408, 2001.

24. Makhija R and Kingsnorth AN: Cytokine storm in acute pancreatitis. J Hepatobiliary Pancreat Surg 9: 401-410, 2002.

25. Yu J, Deng W, Wang W, Ding Y, Jin H, Chen C, Chen X, Xiong X and $\mathrm{Xu}$ S: Inhibition of poly (ADP-ribose) polymerase attenuates acute kidney injury in sodium taurocholate-induced acute pancreatitis in rats. Pancreas 41: 1299-1305, 2012.

26. Chuang PY and He JC: JAK/STAT signaling in renal diseases. Kidney Int 78: 231-234, 2010.

27. Kuratsune M, Masaki T, Hirai T, Kiribayashi K, Yokoyama Y, Arakawa T, Yorioka N and Kohno N: Signal transducer and activator of transcription 3 involvement in the development of renal interstitial fibrosis after unilateral ureteral obstruction. Nephrology (Carlton) 12: 565-571, 2007.

28. Berthier CC, Zhang H, Schin M, Henger A, Nelson RG, Yee B, Boucherot A, Neusser MA, Cohen CD, Carter-Su C, et al: Enhanced expression of janus kinase-signal transducer and activator of transcription pathway members in human diabetic nephropathy. Diabetes 58: 469-477, 2009.

29. Lu TC, Wang ZH, Feng X, Chuang PY, Fang W, Shen Y, Levy DE, Xiong H, Chen N and He JC: Knockdown of Stat3 activity in vivo prevents diabetic glomerulopathy. Kidney Int 76: 63-71, 2009.

30. Yu JH, Kim KH and Kim H: Suppression of IL-1beta expression by the Jak 2 inhibitor AG490 in cerulein-stimulated pancreatic acinar cells. Biochem Pharmacol 72: 1555-1562, 2006.

31. Duan W, Yang Y, Yan J, Yu S, Liu J, Zhou J, Zhang J, Jin Z and Yi D: The effects of curcumin post-treatment against myocardial ischemia and reperfusion by activation of the JAK2/STAT3 signaling pathway. Basic Res Cardiol 107: 263, 2012. 